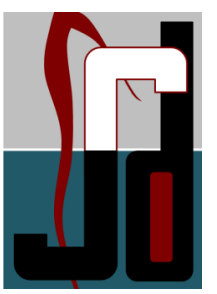

GASE REPORT

\title{
A Cephalometric Technique to Aid in the Positioning of a Dental Implant
}

Sibu Sajjan Simon ${ }^{1}$, Ranu Kumari A², Arun Paul Charllu³ , Sajani Ramachandran ${ }^{4}$

ABSTRACT: Dental implants have become a viable choice in treatment planning to replace missing teeth. Case selection in regards to various factors is of utmost importance to have a predictable outcome when considering treatment with dental implants. Advanced diagnostic techniques, using sophisticated software have become invaluable aids in predicting the locations for dental implants. Often the cost and accessibility to such advanced techniques deprive the patients of having a dental implant placed with superior techniques. This article aims to highlight a simple and modified technique using a lateral cephalometric radiograph, which was used to identify the path of implant placement, in a region with unique limitations.

Key-words: cephalometric radiograph, surgical guide, dental implant

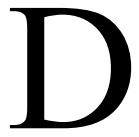

entistry as a service relies on precision for the quality of its outcomes. Implant dentistry requires a high degree of precision from the stage of planning to the final stages of the restoration. Precision can only be translated into manual dexterity with an accurate visualisation of the area of operation. Advanced technology especially in the field of dental imaging is changing the scenario of treatment planning in all the areas of dentistry. Today, advanced imaging techniques assisted by appropriate hardware and software can help to successfully generate a view of the final implant position. $^{[1,2]}$
Devices using the recent advances in diagnostic imaging technology are often expensive and far from reach in remote areas. This case report highlights a simple modification that was made to enhance a two dimensional view provided by a lateral cephalogram to enable placement of a dental implant to replace the missing left maxillary lateral incisor, that was complicated by the presence of a narrow space.

\section{CASE REPORT:}

An eighteen year old boy came to the Department of Dental Surgery, Christian Medical College and Hospital, Vellore, India, with a chief complaint to 
replace his missing left maxillary lateral incisor.

He claimed that he had apparently lost his deciduous teeth in the same region before 2 years. Upon initial clinical examination it was found that the patients complaint concerning his missing left maxillary lateral incisor was legitimate and a routine intra oral periapical radiograph of the region revealed a clear space of approximately 6 $\mathrm{mm}$ between the roots of the adjacent central incisor and canine[Fig 1].

A detailed case history was taken. The investigations revealed that the total mesiodistal space in the region of the missing lateral incisor was $6 \mathrm{~mm}$, with adequate length in the cervico apical region as compared to the roots of the adjacent teeth. The ridge mapping procedure revealed that there was adequate bone in the buccolingual dimension to accommodate a mini implant with a diameter of $3 \mathrm{~mm}$ diameter. Since the socioeconomic status of the patient proved a limitation for using other advanced diagnostic

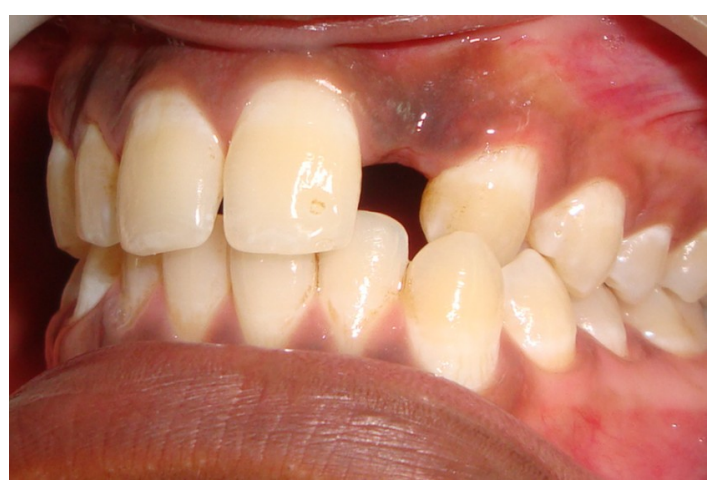

Fig-1: Missing Left maxillary lateral incisor techniques for implant placement, a modified lateral cephalometric view was taken in addition to the other investigations.[Fig 2]

The modification was made possible by fabricating an occlusion rim in the region missing maxillary left lateral incisor region and placing a custom made L shaped 21 gauge wire in the space of the missing teeth. The $\mathrm{L}$ shape of the wire was initially angulated using the long axis of the adjacent teeth and the inclination of the labial alveolar mucosa and the temporary splint was placed in the maxillary arch to facilitate a lateral Cephalometric radiograph ${ }^{[3]}[$ Fig 3]. The resulting image was used to align the $\mathrm{L}$ shaped 21 gauge wire to make it lie parallel with the angle formed by the labial alveolar bone in the region of the maxillary left lateral incisor. A second image was then made to confirm that the angle formed by the L shaped wire was coinciding with the angle formed by labial surface of the alveolar bone in the region of the maxillary left lateral incisor. The

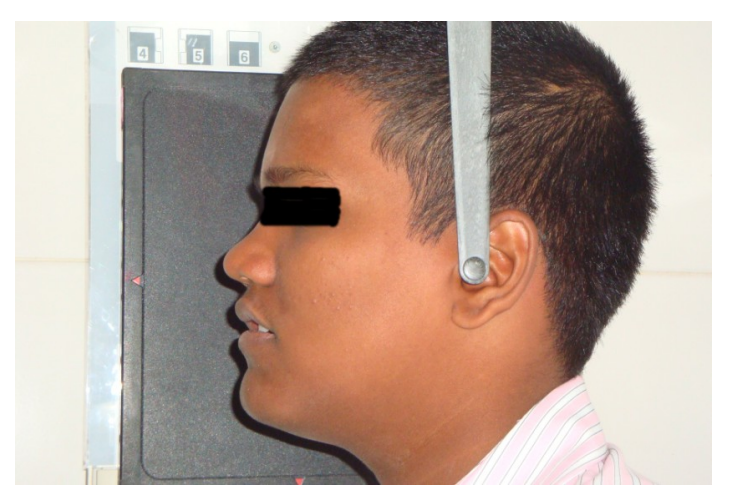

Fig- 2: Positioning patient for a lateral Cephalogram 
occlusion rim was then converted to a surgical template ${ }^{[4]}$ with the final $\mathrm{L}$ shaped wire embedded into the labial surface adjacent to the missing maxillary lateral incisor.

A surgical protocol was formulated and the patient was prepared for surgery. The surgical guide was checked for its accuracy. An open technique was used to expose the site of implant placement. After securing the raised flap and levelling the alveolar crest, a pilot hole was placed with the assistance of the surgical guide using a round bur[Fig 4]. This was followed by an osteotomy with the aid of a $2 \mathrm{~mm}$ drill, to a depth of $10 \mathrm{~mm}$. An intraoperative periapical view helped to confirm that the surgical phase was proceeding as planned in the treatment planning phase [Fig 5].The mini implant with a platform of 3 $\mathrm{mm}$ in diameter and $10 \mathrm{~mm}$ in length (Life Care Devices Pvt Ltd) was placed in the prepared bone in the region of the missing maxillary left lateral incisor[Fig 6]. The surgical site was closed appropriately after ensuring the primary stability of the mini implant and the patient was given the necessary postoperative instruction and care.

An intraoral periapical view confirmed the position of the implant in the region of the maxillary left lateral incisor [Fig 7]. In the immediate postoperative phase, a temporary crown was planned as part of the treatment plan. The

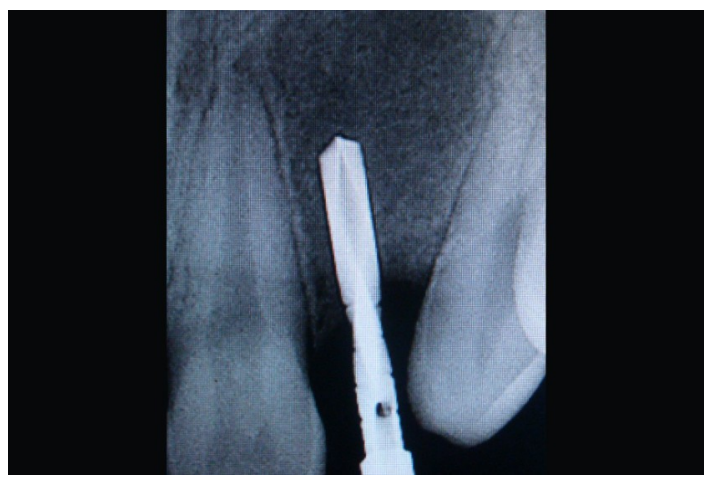

Fig- 5 : Surgical drill in position

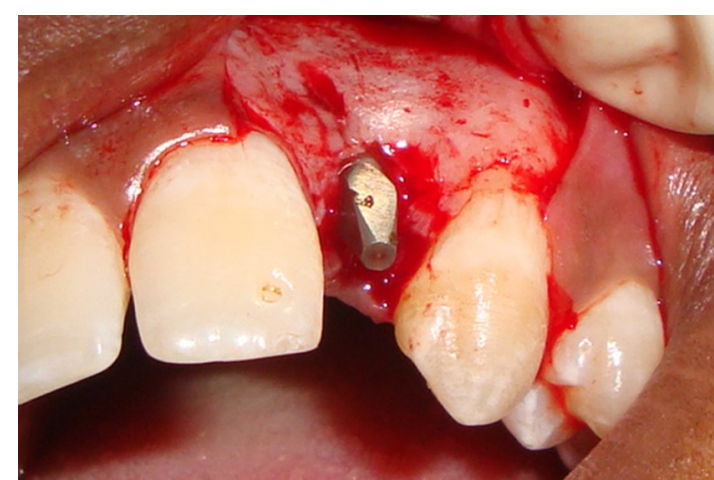

Fig- 6: Mini Implant successfully placed into the narrow space 


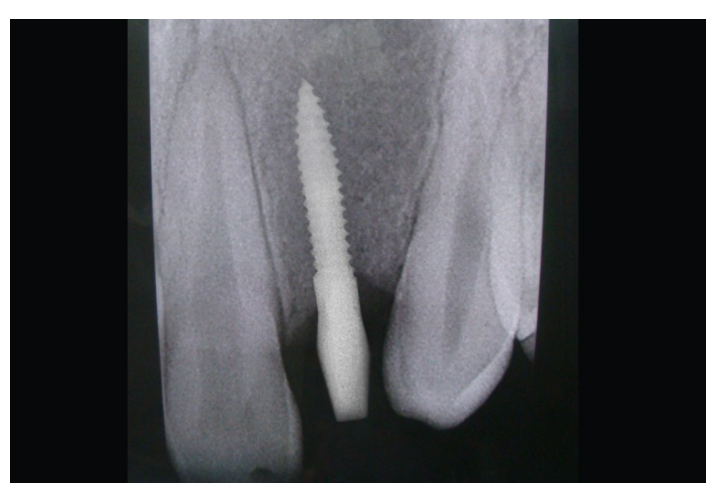

Fig- 7: Intraoral periapical view of the implant

patient was placed on regular review appointments till the fabrication of the final restoration. The modified technique using the cephalogram in addition to the other diagnostic technique proved to be an approximate guide to the surgical procedure.

\section{DISCUSSION:}

Recent advances in imaging techniques have increased the level of precision that can be delivered at the treatment phase for dental implants ${ }^{[5,6]}$. The paradigm shift in imaging technology has made diagnostic techniques more affordable and accessible. In the past, imaging data from Cephalometric radiographs have been an invaluable aid to provide diagnostic information in treatment planning with dental implants. Engleman et al describes a technique of using a stent with the making of a computed tomography ${ }^{[7]}$. The described case report describes a similar technique of using a stent with a lateral cephalogram.

$$
\text { Belatro et al used the }
$$
cephalometric analysis in addition to other techniques in treatment planning for dental implants. ${ }^{[8]}$ Yasunori used the imaging data from a lateral cephalogram to place implant in a patient with an edentulous maxilla with a severe class III intermaxillary relationship. ${ }^{[9]}$ Divya et al have extensively documented the use of lateral cephalometry in imaging for dental implants. ${ }^{[10]}$

The presented case report proved to be challenging in regard to the narrow space and the socioeconomic concerns. In this case a regular lateral cephalometric radiograph was modified by using an occlusion rim with an embedded L shaped 21 gauge wire. The temporary stent was then converted to a surgical stent and was used effectively to facilitate the placement of the mini implant (Life Care Devices Pvt Ltd) in the narrow space. Although the significance from the resulting radiograph was minor, it helped to add an additional dimensional view to the existing two dimensional view provided by the intra oral periapical view.

Though the described technique was used to successfully predict the labio-palatal 
inclination of the proposed implant of the anterior regions by adding an additional dimension to the two dimensional view provided by an intraoral periapical view, it could be further improvised and standardized to be used with other advanced diagnostic techniques for a predictable outcome.

\section{REFERENCES:}

1. Mallya SM, Potluri A. Diagnostic imaging for implants. J Indian Aca Oral Med Radiol 2004; $16: 174-80$.

2. Carl E Misch: Contemporary Implant Dentistry. 3rd ed. Mosby. 2008.

3. SotiriosTetradis, Mel L Kantor. Extraoral Radiographic Examinations. In: Staurt C White, Michael J Pharoah, editors. Oral Radiology. Principles and Interpretation. Ed 6. Mosby: 2009. 191-206.

4. Pesun IJ, Gardner FM: Fabrication of a guide for radiographic evaluation and surgical placement of implants. J Prosthet Dent. 1995 June,
$73(6): 548-52$.

5. Chan HL, Misch K, Wang HL: Dental imaging in implant treatment planning. Implant Dent.2010 Aug;19(4):288-98.

6. Angelopoulos C, Aghaloo T : Imaging technology in implant diagnosis. Dent ClinNorth Am. 2011 Jan; 55 (1):141-58.

7. Engelman MJ, Sorensen JA, Moy P. Optimum placement of osseointegrated implants. J Prosthet Dent. 1988;59(4):467-73.

8. GC Beltrao, AT de Abreu, RG Beltrao, NF Finco: Lateral cephalometric radiograph for the planning of maxillary implant reconstruction; DentomaxillofacRadiol. 2007 Jan; 36(1): 45-50.

9. Hotta Y: Use of cephalometric analysis for implant placement in a patient with an edentulous maxilla with a severe class III intermaxillary relationship. J Oral Implantol. 2004; 30 (1): 7-13.
Address for correspondence:

Dr.Sibu Sajjan Simon

Assistant Professor,

Christian Medical College and Hospital,

Vellore

simonprostho@gmail.com

Authors:
${ }_{1,3}$ Assistant Professor, Christian Medical College and
Hospital, Vellore
${ }^{2}$ Professor, MGPGI, Puducherry
${ }^{4}$ Associate Professor, PIMS, Puducherry

Authors:

Hospital, Vellore

${ }^{4}$ Associate Professor, PIMS, Puducherry

How to cite this article:

Sibu Sajjan Simon, Ranu Kumari A, Arun Paul Charllu, Sajani Ramachandran. A Cephalometric Technique to Aid in the Positioning of a Dental Implant . Journal of Scientific Dentistry 2013;3(1):20-24

Source of Support: Nil, Conflict of Interest: None declared 